w domie, w domu, w domu, ut dip? -40 do $w d$ w dow

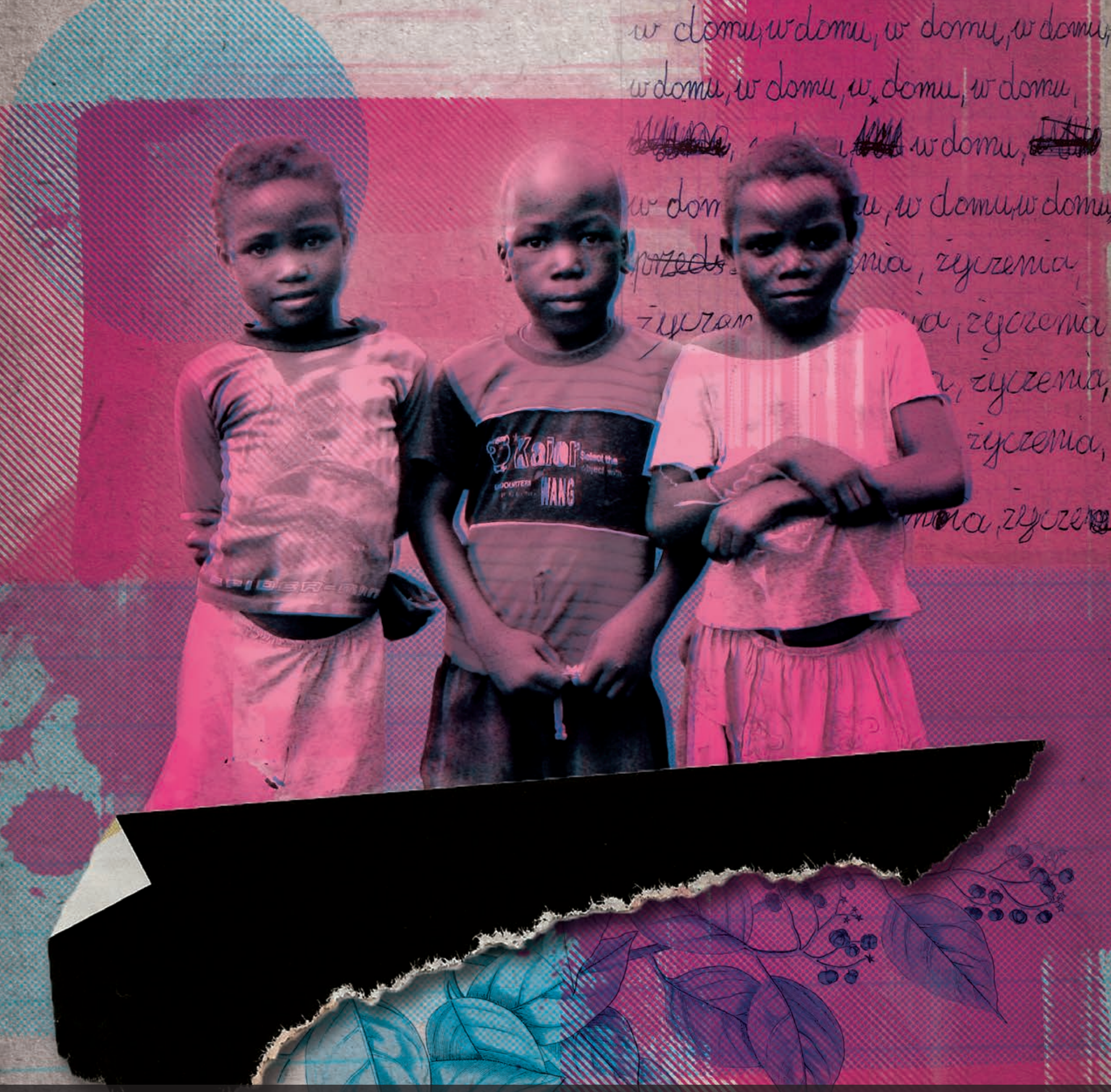

LA INVESTIGACIÓN ACCIÓN Y EL APRENDIZAJE POR PROYECTOS EN EL MARCO DEL MODELO PEDAGÓGICO ENSEÑANZA PARA LA COMPRENSIÓN. EXPERIENCIA DEL COLEGIO VISIÓN MUNDIAL EN COMUNIDADES VULNERABLES DE MONTERÍA. 


\title{
LA INVESTIGACIÓN ACCIÓN Y EL APRENDIZAJE POR PROYECTOS EN EL MARCO DEL MODELO PEDAGÓGICO ENSEÑANZA PARA LA COMPRENSIÓN. EXPERIENCIA DEL COLEGIO VISIÓN MUNDIAL EN COMUNIDADES VULNERABLES DE MONTERÍA. \\ (c)

\author{
Action research and Project Based Learning in Pedagogical teaching for understanding model \\ framework. "Visión Mundial" school in Monteria's vulnerable communities experience
}

A pesquisa ação e o aprendizado por projetos no marco do modelo pedagógico de ensino para a compreensão. Experiência do colégio Visão Mundial em comunidades vulneráveis de Montería.

RECEPCIÓN:27DEJUNIODE 2015

Martha Chaves Silva (Colombia)

Magíster en Educación

machsi7@gmail.com

Universidad de Córdoba

\section{es}

\section{RESUMEN}

Este artículo presenta resultados derivados de "La investigación acción y el aprendizaje por proyectos en el marco del modelo pedagógico Enseñanza para la comprensión", en la Maestría de Educación en la Universidad de Córdoba - SUE-CARIBE1. E1 texto tiene como propósito mostrar la incidencia de la investigación acción y el modelo pedagógico Enseñanza para la comprensión en la calidad educativa, con comunidades en condiciones de pobreza y vulnerabilidad, en el Colegio Visión Mundial de Montería, Córdoba. La metodología se sustenta en el modelo de la investigación-acción (IA), que posibilita a los participantes actuar de manera reflexiva y colectiva sobre los problemas que los aquejan, con el fin de transformarlos y transformarse. $\mathrm{El}$ análisis de los resultados demuestra que la educación de calidad para poblaciones en condiciones de pobreza es no solo necesaria, sino posible, aminorando la fuerte estratificación que persiste en el sistema educativo local y nacional.

PALABRAS CLAVE: investigación acción, enseñanza para la comprensión, proyectos de aula, prácticas pedagógicas, comunidades vulnerables
EVALUACIÓN:4DE SEPTIEMBREDE 2017

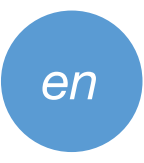

ABSTRACT

This article presents results from "Action research and Project Based Learning in Pedagogical Teaching for Understanding Model framework", in Education Master at the University of Córdoba - SUE-CARIBE2. The purpose of the document is to show action research and pedagogical teaching for understanding model incidence in educational quality, within communities in conditions of poverty and vulnerability, at the "Visión Mundial school of Montería, Córdoba. Methodology is based on action research model, which enables participants to act reflexively and collectively on the problems that afflict them, in order to transform it and transform them. Analysis of the results shows that quality education for populations living in poverty is not only necessary, but also possible, reducing the strong stratification that persists both in local and national education system.

KEYWORDS: action research, teaching for understanding, classroom projects, pedagogical practices, vulnerable communities
ACEPTACIÓN:4DE DICIEMBREDE 2017

Lilia Barrios Oviedo (Colombia) Magíster en Educación limarbao@gmail.com
Universidad de Córdoba

\section{RESUMO}

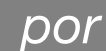

Este artigo apresenta resultados derivados de "A pesquisa ação e o aprendizado por projetos no marco do Modelo Pedagógico Ensino para a Compreensão", no mestrado de Educação na Universidade de Córdoba - SUE-CARIBE3. $\mathrm{O}$ texto tem como propósito mostrar a incidência da pesquisa ação e o modelo pedagógico ensino para a compreensão na qualidade educativa, com comunidades em condições de pobreza e vulnerabilidade, no Colégio Visão Mundial de Montería, Córdoba. A metodologia se sustenta no modelo da pesquisa-ação (IA), que possibilita aos participantes atuar de maneira reflexiva e coletiva sobre os problemas que os aqueixam, com o fim de transformá-los e se transformar. A análise dos resultados demostra que a educação de qualidade para populações em condições de pobreza é não só necessária, mas possível, diminuindo a forte estratificação que persiste no sistema educativo local e nacional.

PALAVRAS CHAVE: ansiedad; depresión; asertividad; regulación emocional; niñas y jóvenes en situación de desamparo..

\section{PARA CITAR ESTE ARTÍCULO/TO CITE THIS ARTICLE/PARA CITAR ESTE ARTIGO:}

Chaves Silva, M. y Barrios Oviedo, L., (2017). La investigación acción y el aprendizaje por proyectos en el marco del modelo pedagógico Enseñanza para la comprensión. Experiencia del colegio Visión Mundial en comunidades vulnerables de Montería. Panorama, 11(21), 29-51.

1 Universidades Estatales del Caribe Colombiano, con estrategia de formación avanzada para educadores de excelencia, que participan en la formulación e implementación de las políticas educativas desde la región Caribe, con trascendencia nacional y hacia la internacionalización.
2 State Universities of the Colombian Caribbean, with advanced training strategy for excellence educators, who participate in Caribbean region educational policies formulation and implementation, with national transcendence and internationalization.

\footnotetext{
3 Universidades Públicas do Caribe Colombiano, com estratégia de formação avançada para educadores de excelência, que participam na formulação e implementação das políticas educativas desde a região Caribe, com transcendência nacional e dirigida para a internacionalização.
} 


\section{INTRODUCCIÓN}

El departamento de Córdoba, como la mayoría de los departamentos del Caribe colombiano, posee ciertas particularidades tanto en lo social como en lo económico, político, ambiental y cultural. Entre estas, especialmente en el aspecto social, desde hace varios años, se han evidenciado graves problemas de violencia, sobre todo en el área rural. Las poblaciones han llegado a ser víctimas del desplazamiento, y en su proceso de reubicación deben afrontar desafíos de superación personal en situaciones adversas; por ejemplo, de acomodo en un nuevo territorio que no ofrece las garantías a sus necesidades, tales como educación, salud, vivienda, transporte, servicios públicos, entre otras.

Según Negrete (2007), estos procesos migratorios han hecho aumentar la población en ciudades como Montería, capital del departamento de Córdoba, con más de cuarenta asentamientos subnormales, todos receptores de una población desplazada de aproximadamente 124.484 inmigrantes, como consecuencia de la violencia política y económica, contribuyendo de esta manera a crear una sociedad que se debate en medio de la pobreza y la inequidad, aumentando las desigualdades, la desesperanza y el sentimiento de injusticia.

Este problema se ha ido incrementando en la ciudad, tanto así que los niños, niñas, adolescentes y jóvenes vinculados al sistema educativo están a menudo en la primera línea de los conflictos violentos, haciéndolos crecer en condiciones de alta vulnerabilidad e impidiéndoles que desarrollen sus potenciales como sujetos sociales.

Este estudio se desarrolló en el colegio Visión Mundial, de propiedad de la ONG World Vision Colombia, en la ciudad de Montería, con cinco directivos, ocho adminis-

Panorama | trativos, 38 docentes y 789 estudiantes desde el pre-espp. 39-51 colar hasta la media técnica en sistemas, provenientes Volumen 11 de las comunidades más pobres de las comunas 4, 5 y Número 21 6, las cuales están conformadas, mayoritariamente, por personas desplazadas por la violencia, lo que las ubica en condiciones de alta vulnerabilidad e inequidad.

Para lo anterior, se tuvieron en cuenta consideraciones teóricas y conceptuales con respecto a la investigación acción y las estrategias pedagógicas y didácticas, en el marco de los proyectos de aula y el modelo pedagógico Enseñanza para la comprensión.

Metodológicamente, se asume desde un enfoque cualitativo interpretativo, con base en el método de investigación acción (IA), que involucra a diversos agentes de la comunidad educativa, teniendo en cuenta los espacios académicos, administrativos y de desarrollo comunitario, inmersos en el proceso de enseñanza y aprendizaje, dentro y fuera de la escuela pues, la IA es una forma de indagación introspectiva colectiva emprendida por participantes en situaciones sociales con el objeto de mejorar la racionalidad y la justicia de sus prácticas sociales o educativas, así como su comprensión de esas prácticas $\mathrm{y}$ de las situaciones en que estas tienen lugar (Kemmis, S. y McTaggart, 1992), con un enfoque cualitativo, ya que está motivada por búsquedas y no por la pretensión de comprobar verdades. Desde esta perspectiva, Pérez Serrano (1994) afirma que la investigación cualitativa es considerada "como un proceso activo, sistemático y riguroso de indagación dirigida, en la que se toman decisiones sobre lo investigable en tanto se está en el campo de estudio" (p. 46).

Los resultados han permitido evidenciar que es posible el desarrollo social inclusivo, a partir de un trabajo reflexivo, colectivo y participativo, con prácticas pedagógicas exitosas y replicables, a partir de la investigación acción y el aprendizaje por proyectos en el marco del modelo pedagógico Enseñanza para la comprensión, en comunidades educativas de alta vulnerabilidad.

\section{MARCO TEÓRICO}

El modelo Enseñanza para la comprensión como apuesta para la transformación educativa y social.

¿Qué es la comprensión? ¿Cómo enseñar para la comprensión? ¿Cómo pueden los estudiantes aprender para comprender? ¿Cuáles podrían ser los programas de estudio, las actividades, y las evaluaciones que les darían, a diario, el mejor apoyo a la enseñanza para la comprensión? ¿Cómo podrá la formación construir una comprensión profunda tanto de los contenidos disciplinares como de la complejidad de la tarea de enseñar en las instituciones educativas? Ante estos inminentes cuestionamientos que día a día afrontan los maestros en los procesos de enseñanza y aprendizaje, es necesario analizar las implicaciones teóricas y metodológicas del 
modelo Enseñanza para la comprensión como alternativa para la transformación educativa y social.

El sentido de la enseñanza para la comprensión

Una de las preocupaciones centrales que desde los ámbitos académicos se ha gestado, tiene que ver con la concepción de nuevos paradigmas educativos que contribuyan a la generación de una sociedad cada vez más crítica, reflexiva y constructiva. La delgada línea que divide el proceso de enseñanza y aprendizaje cuestiona la brecha existente entre la teoría y la práctica del accionar pedagógico. Como lo afirma David Perkins (1995):

... no es que no sepamos lo suficiente como para tener escuelas en las que un gran número de personas con diferentes capacidades, intereses y provenientes de medios socioculturales y familiares diferentes puedan aprender. El problema es que más allá de los desarrollos acerca del aprendizaje, las investigaciones sobre las escuelas eficaces, los estudios sobre las posibilidades del cambio y la innovación en educación, es muy complejo el salto entre la enunciación de nuestros saberes y el "uso activo" de ellos (p. 26).

Ahora bien, en los últimos cincuenta años, investigaciones relacionadas con la consolidación de escenarios que contribuyan a la generación de aprendizajes significativos y genuinos han tomado papel protagónico en las esferas académicas, desarrollándose en el marco de estas preocupaciones el Proyecto Zero de la Universidad de Harvard, el cual fue fruto del trabajo colaborativo de investigadores y docentes que se enfocaron en los aportes teóricos de David Perkins, Nelson Goodman, Howard Gardner, Vito Perrone, J. Bruner, R.F. Elmore, M.W. McLaughlin, entre muchos más.

Este proyecto educativo, titulado Enseñanza para la comprensión, brinda una propuesta de diseño para el trabajo en las aulas y en las escuelas que, si bien propone un modelo de planificación, encierra en él una lógica de concepción acerca de la enseñanza, el aprendizaje y una postura ética sobre la certeza de que todos somos capaces de comprender y que, además, se puede ayudar a que esto sea posible por medio de una enseñanza pertinente.
Paula Pogré (2007) reflexiona sobre los aportes que ha dejado el modelo Enseñanza para la comprensión, resaltando los siguientes aspectos:

- Construir una propuesta didáctica que hace real el trabajo constructivo en las aulas.

- Tener un código común, en un lenguaje claro y sencillo, que facilita la comunicación y el intercambio de experiencias.

- Articular los diferentes componentes de la agenda tradicional de la didáctica: objetivos, contenidos, actividades y evaluación, en una propuesta comprensiva que permite acercar finalmente nuestras teorías y nuestras prácticas.

- Darnos herramientas para hacer realidad el deseo de una genuina educación de calidad para todos en dos sentidos: educar para la diversidad social, educar respetando las diferencias individuales.

- Enseñar integrando el pensar, el sentir y el actuar.

- Educar para integrar el pensar, el sentir y el actuar.

Todos estos aspectos reafirman la trascendencia del modelo Enseñanza para la comprensión, el cual promueve el desarrollo de una inteligencia compartida que permite crear y sostener culturas de comprensión (Perkins, 1995).

Pero ¿qué es comprender? Según Perkins (1999), “es la habilidad de pensar y actuar con flexibilidad a partir de lo que uno sabe..." (p. 1). Es decir, recorrer los caminos del conocimiento mediante el apoyo mancomunado de experiencias previas y análisis crítico de la realidad social. Es vislumbrar la frontera de lo desconocido por medio de aprendizajes significativos.

Por otra parte, el modelo Enseñanza para la comprensión ha sido recreado y utilizado para la enseñanza en todos los niveles: desde el ciclo inicial hasta la educación superior y en la formación docente en países diversos, convirtiéndose hoy en un interesante dispositivo que permite que educadores de diferentes niveles y regiones del mundo reflexionen colaborativamente sobre la enseñanza.

En primera instancia, hay que tener en cuenta que dentro de este marco, la Comprensión desarrolla Tópicos Generativos, es decir se consolida mediante el uso reflexivo de conceptos, ideas, temas relativos a una disciplina o campo de conocimiento, que tienen ciertas características que los hacen especialmente indicados
La investigación

acción y el

aprendizaje por

proyectos en el

marco del modelo pedagógico

Enseñanza para

la comprensión.

Experiencia del

colegio Visión

Mundial en

comunidades

vulnerables de

Montería. 
Martha Chaves

Silva |

Lilia Barrios Oviedo

Por otra parte, el Modelo desarrolla Metas de Comprensión, es decir, permite la identificación de conceptos, procesos y habilidades que queremos que los alumnos desarrollen. Además, enfocan aspectos centrales del tópico generativo, identificando lo que consideramos más importante que nuestros alumnos comprendan sobre él.

De igual forma, la Comprensión es un Desempeño, es decir, la capacidad de relacionar, operar, describir, comparar, diferenciar, adecuar, relatar, diagramar, analizar, decidir, representar, secuenciar, organizar, extrapolar, etc. Son actividades que van más allá de la memorización de conocimientos y habilidades rutinarias. En estas actividades los alumnos reconfiguran, expanden y aplican lo que han aprendido al mismo tiempo que exploran y construyen nuevos aprendizajes a partir de los previos. Ayudan tanto a construir como a demostrar la comprensión.

La Comprensión es Evaluación Continua, por consiguiente, es el proceso de brindar sistemáticamente a los alumnos una respuesta clara sobre su trabajo, contribuyendo a mejorar sus desempeños de comprensión. Este proceso exige que los desempeños estén guiados por criterios de evaluación que sean claros, públicos, relacionados con las metas de comprensión y orientados por los hilos conductores.

Finalmente, la Enseñanza para la comprensión ofrece un rango amplio de ideas concretas para la planeación: secuenciar los desempeños de comprensión de manera que se vaya construyendo de lo más sencillo a desempeños culminatorios más complejos... o la utilización constante de herramientas de valoración continua que provean retroalimentación y apoyo a los aprendices durante todo el proceso (Perkins, 1999).
LOS PROYECTOS DE AULA COMO ESTRATEGIA DIDÁCTICA INTEGRADORA

El aula se considera como el espacio escolar creado para construir conocimiento y establecer procesos de enseñanza y aprendizaje; es un lugar de convivencia e intercambio educativo entre los estudiantes y el docente. En la antigüedad, se consideraba como un palacio del príncipe soberano, en educación, un lugar de respeto y de reflexión.

Es en el aula donde el docente y sus estudiantes generan un contrato pedagógico que cumplen ambas partes en pos de construir experiencias en grupo, respetando las condiciones, compartiendo sentimientos de solidaridad y colaboración, un lugar que se convierte en laboratorio o taller; en algunas ocasiones estos espacios son móviles y su entorno transforma el ambiente.

En lo general, se reconoce el aula como espacio de producción y reproducción de contenidos ideológicos, culturales, relaciones sociales que lo crean y lo mantienen. En este sentido, se puede pensar el aula como un espacio donde se juega un orden social y cultural, así como diversas manifestaciones de su oposición (Guzmán y Jiménez, 1991, p. 338).

Desde esta perspectiva, el conocimiento se construye de manera activa por quien aprende mediante la interacción con otros en un medio social, y el aprendiz puede elaborar personalmente lo aprendido, logrando con ello que el aprendizaje le sea funcional y significativo (Denegri, 1996; Denegri y Martínez, 2001). Ello implica que el alumno se enfrenta a los nuevos contenidos con una serie de conceptos, representaciones y conocimientos ya adquiridos y teorías propias que le permiten explicarse el mundo.

Estos preconceptos incluyen también actitudes, motivaciones, expectativas y atribuciones relacionadas con sus experiencias de vida; ya no se trata de una transmisión de contenidos sino de una construcción conjunta donde el profesor, desde su rol de experto y a la vez de facilitador, aporta las herramientas para que el alumno establezca relaciones, pero es este último quien finalmente construye su propio conocimiento (Denegri, 2000). 
Dentro de este marco, se promueve un cambio cualitativo en la forma de ver al mundo, en la forma de hacer las cosas, en la forma de enseñar. Es un cambio entendido como parte de la dinámica individual y grupal, es un nuevo desarrollo escolar que restablece el carácter de transformación social y cultural que tiene la escuela, que constituye también una forma de organizar sistemáticamente el aprendizaje y la enseñanza, involucrando directamente a los actores del proceso, integrando y correlacionando áreas del conocimiento, logrando que todos y cada uno se desenvuelvan, adecuándose desde el currículo a lo planeado y ejecutado.

Por su parte, el estudiante debe ser capaz de establecer relaciones sustantivas entre lo que ya sabe y los nuevos conocimientos que está construyendo; ello implica modificar los esquemas de conocimiento que ya posee para construir esquemas de mayor complejidad. El logro de aprendizajes significativos requiere de una intensa actividad mental, mientras que el docente estimula el conflicto cognitivo mediante actividades que obliguen a la reflexión sobre la acción física y mental.

En este sentido, los proyectos de aula en el proceso de enseñanza y aprendizaje son un proceso fundamentalmente interactivo, en los que es importante la relación maestro-alumno, como la que establecen los alumnos entre sí. Por ello, los proyectos de aula favorecen el intercambio de información, la confrontación de puntos de vista distintos, y en esta actividad el profesor debe estar especialmente atento a las interacciones que se producen en el grupo, interviniendo para propiciar que se analicen y resuelvan los conflictos en un clima de aceptación, ayuda mutua, cooperación y tolerancia. Esto involucra favorecer la autonomía de los escolares en la toma de decisiones, asumiendo sus responsabilidades como miembros de un grupo. Desde esta mirada, el aprendizaje significativo cobra gran importancia y el proyecto, una alternativa novedosa donde el estudiante genera la construcción de conocimiento en una amplia gama de situaciones y circunstancias (Coll, 1981).

Por su parte, Sayago Z. (2003) indica, igualmente, que los proyectos de aula desencadenan procesos de cuestionamientos e identificación de problemas de una manera entretenida, generados por dos fuentes: una parte, los estudiantes, por su inherente curiosidad, el saber y el deseo de construir o experimentar; la otra, las propuestas expuestas por el docente mediante actividades previamente organizadas, originando una serie de interrogantes que conlleven a la comprensión y aprendizaje en los educandos; además, permiten la globalización e integración de los aprendizajes y favorecen el aprendizaje significativo.

Mediante el desarrollo de los proyectos de aula, los alumnos asimilan y atribuyen significado a los contenidos propuestos; para ello establecen las relaciones entre los conocimientos previos que ya poseen y los contenidos nuevos, objeto del aprendizaje. Al respecto, la función primordial de los proyectos es proporcionar calidad educativa a los educadores, educandos, padres y otros miembros de la comunidad, a fin de garantizar la equidad, adecuándolos a las necesidades específicas del entorno social.

En efecto, los proyectos de aula según Hernández y Ventura (1994), incorporan una actitud integradora de contenidos, se enmarcan en la explicativa de la globalización como sumatoria de asignaturas, interdisciplinariedad y estructura de aprendizaje. Sin embargo, la concepción de proyecto integrador exige un conjunto de saberes que pueden darse en forma simultánea o sucesiva, su desarrollo es responsabilidad del colectivo docente, motivado por los directivos académicos, intervención de los padres y madres de familia y demás miembros de la comunidad educativa.

De igual forma, este sistema se convierte en una unidad integradora, donde la docencia, la investigación y la práctica real son elementos básicos en el proceso educativo: se realiza una combinación entre la práctica y la teoría. Garantiza un ejercicio integral y permanente, desarrollado desde las mallas curriculares hacia el proyecto de aula, que contiene posibilidades de diversas líneas de investigación y están enfocados hacia la innovación pedagógica, donde se integra la enseñanza y la investigación a partir de la identificación de los objetos de transformación y de los procesos de aprendizaje, creando vínculos entre la institución, el estudiante, el docente, la familia y la realidad del contexto social.

En torno a la evaluación del proyecto integrador, el resultado final del aprendizaje es un proceso de reflexión de los objetivos propuestos, considerando el pensamiento cualitativo, la iniciativa teórica, la creatividad de diversas situaciones en escenarios de participación y construcciones significativas de contenidos culturales,
| La investigación

acción y el

aprendizaje por

proyectos en el

marco del modelo

pedagógico

Enseñanza para

la comprensión.

Experiencia del

colegio Visión

Mundial en

comunidades

vulnerables de

Montería.
| Panorama

| pp.39-51

| Volumen 11

| Número 21

| Julio-Diciembre

| 2017

| 43 
mediante la cooperación e interacción que establecen con el docente, compañeros y diferentes miembros de la comunidad escolar.

Martha Chaves Silva |

Lilia Barrios Oviedo |

Esta investigación es de tipo cualitativo, sustentada en el método investigación acción (IA), ya que está motivada por búsquedas y no por la pretensión de comprobar verdades; tampoco le asiste la certidumbre, sino la inquietud de construir una significación acerca de una realidad, posibilitando a los participantes actuar de manera reflexiva y colectiva sobre los problemas que los aquejan, donde los procesos educativos tienen como función trascendental la transformación humana. En este sentido, la IA es una forma de indagación introspectiva colectiva con el objeto de mejorar la racionalidad y la justicia de sus prácticas sociales o educativas, así como la comprensión de esas prácticas y de las situaciones en que estas tienen lugar (Kemmis y McTaggart, 1992).

Desde esta perspectiva, Pérez (1994) afirma que la investigación cualitativa es considerada "como un proceso activo, sistemático y riguroso de indagación dirigida, en la que se toman decisiones sobre lo investigable, en tanto se está en el campo de estudio" (p. 46).

El término investigación acción, IA, surge del autor Kurt Lewis (1944), el cual describía una forma de investigación que podía ligar el enfoque experimental de la ciencia social con programas de acción social que respondiera a los problemas sociales principales de entonces, mediante la investigación acción, argumentando que se podía lograr en forma simultáneas avances teóricos y cambios sociales (Kemmis y Mctaggart, 1992).

El principal representante de la investigación-acción, desde un enfoque interpretativo, la define como un estudio de una situación social con el fin de mejorar la calidad de la acción dentro de la misma. La entiende como una reflexión sobre las acciones humanas y las situaciones sociales vividas por el profesorado, que tiene como objetivo ampliar la comprensión de los docentes acerca de sus problemas prácticos. Las acciones van encaminadas a modificar la situación, una vez que se logre una comprensión más profunda de los problemas (Elliott, 1993).
Al respecto, Stenhouse (1984) propone integrar en el docente los roles de investigador, observador y maestro, siempre y cuando el profesor ponga en claro que la razón por la cual está desempeñando el rol de investigador es la de desarrollar positivamente su enseñanza y mejorar sus prácticas, en escenarios propiamente humanísticos.

Sobre la base de estos presupuestos conceptuales, se asume que la investigación acción se constituye en un tipo y un método de investigación que compromete de manera consciente y decidida a los actores que en ella participan, convirtiéndolos en sujetos y objetos del proceso, con una actitud de cambio participativa y transformadora.

Por su parte, el enfoque cualitativo como actividad sistémica orientada a la comprensión en profundidad de fenómenos educativos y sociales permite indagar, profundizar y describir a partir de una práctica escolar (Sandín, 2003).

¿Cuáles son las interrelaciones del modelo pedagógico Enseñanza para la comprensión y los proyectos de aula en la comunidad educativa en el marco de la investigación acción?

En este orden de ideas, se identificaron aquellos elementos y rasgos que se constituyeron en fases para el análisis de la investigación. Al respecto, Le Compte (1992) señala que la investigación cualitativa es aquella que extrae descripciones a partir de observaciones que adoptan las formas de entrevistas, grupos de discusión, narraciones, diarios de campo, transcripciones de audio y video, registros escritos de todo tipo.

\section{DISEÑO METODOLÓGICO}

Del colectivo de docentes que laboraban en el colegio Visión Mundial, se conformó la Comisión de evaluación y aprendizaje continuo (CAEC), integrada por ocho maestros, como un proceso cíclico de la investigación sobre problemas individuales y colectivos, la planeación y práctica pedagógica, la reflexión y evaluación continua.

En este sentido, el diseño de esta investigación tomó como base el desarrollo pedagógico y educativo, mediante un proceso de reflexión activo y participativo, 
con el objeto de lograr un mejoramiento continuo de la práctica pedagógica, para la generación de conocimiento y cambio social.

En el esquema que aparece más adelante se sintetiza la operacionalización del diseño metodológico, el cual inicia con el proceso de caracterización que comienza por la interrogación de la realidad respecto a los problemas comunes que afectan a la comunidad educativa. Seguidamente, se avanza hacia la sistematización de las experiencias. Finalmente, se asume la evaluación de las transformaciones alcanzadas para avanzar hacia un nuevo ciclo en el que intervienen los actores de la comunidad educativa. En este caso, la calidad de la educación está unida a la investigación y, al contextualizarla, el docente reflexiona sobre su propia práctica, la transforma, con el propósito de diseñar y aplicar nuevas estrategias y fases dirigidas a mejorar sus logros.

Para la recolección de datos se emplearon y aplicaron entrevistas, grupos de discusión, narraciones, diarios de campo, transcripciones de audio, video y registros escritos de todo tipo incluyendo lo observado. Esta visión de la IA en los procesos escolares propone un modelo cíclico de reflexión-acción-reflexión, en el que se reorganiza la relación entre conocer y hacer, entre sujeto y objeto, configurando y conslidando con cada paso la capacidad de autogestión de los implicados: es un contexto investigativo más abierto y procesual, (Kirchner, 2004; Bru y Basagoiti, 2002).

Figura 1. Diseño de la aplicación del método de investigación acción.

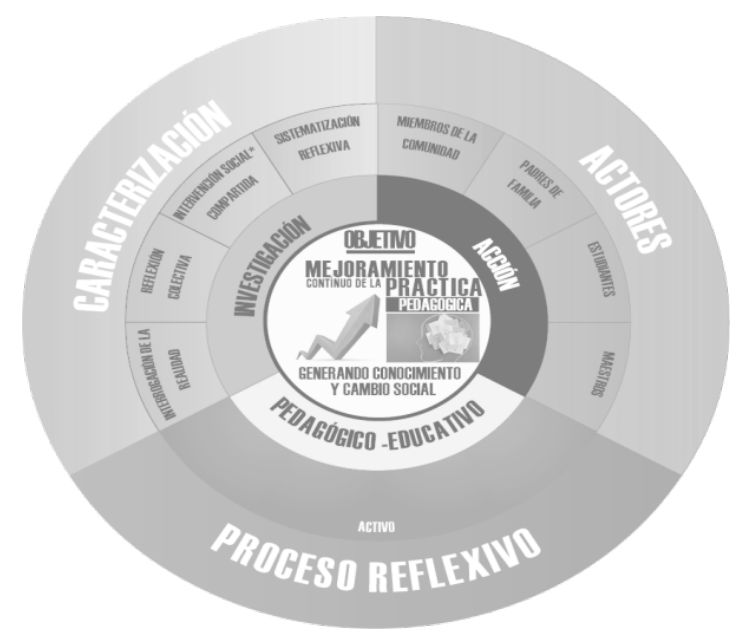

Fuente: elaboración propia.
Para el análisis de los datos e informes recolectados se utilizó la herramienta computacional Atlas Ti; se codificaron y analizaron de forma simultánea para desarrollar conceptos. De igual manera, se siguieron unas reglas de sintaxis que establece el programa y los parámetros que establecieron las investigadoras. Se diseñó una matriz de análisis con las categorías, rasgos, descriptores, microtextos de las entrevistas y comentarios para la relación, comprensión, comparación y contrastación de los hallazgos.

En este sentido, Kaplan (1979) señala que solo se llega a una buena teoría mediante el ejercicio de la imaginación creativa, ya que la formación de una teoría no consiste en descubrir o en destapar un hecho escondido; es un modo de mirar los hechos, organizarlos y representarlos conceptualmente mediante una nueva red de relaciones entre sus partes constituyentes.

\section{FASES DE LA INVESTIGACIÓN}

Las fases de la investigación implican un diagnóstico, la construcción de planes de acción, la ejecución de planes, la reflexión y evaluación continua. A los involucrados les permite redimensionar, reorientar o replantear nuevas acciones y la oportunidad de construir conocimiento. Lewin los denomina ciclos de acción reflexiva: planificación, acción y evaluación de la acción. Por su parte, Kemmis (1992) los incluye en cuatro momentos interrelacionados como planificación, acción, observación y reflexión. Para Pérez Serrano (1998), son pasos o etapas para el acercamiento con la metodología IA: se inician con el diagnóstico de una preocupación temática o problema; luego, la construcción del plan de acción, la puesta en práctica del referido plan y su respectiva observación, la reflexión e interpretación de resultados y la replanificación, si fuera necesaria.

En el siguiente esquema se representan las fases de la investigación, asumidas por las investigadoras, permeadas por una dinámica cíclica de reflexión y evaluación continua, para avanzar en las comprensiones y las acciones mismas hacia niveles cada vez más avanzados, produciendo conocimiento.
La investigación

acción y el

aprendizaje por

proyectos en el

marco del modelo

pedagógico

Enseñanza para

la comprensión.

Experiencia del

colegio Visión

Mundial en

comunidades

vulnerables de

Montería.
| Panorama

| pp. 39-51

| Volumen 11

| Número 21

| Julio-Diciembre

| 2017 
Figura 2. Modelo del método de investigación acción asumido sobre la experiencia.

Martha Chaves

Silva |

Lilia Barrios

Oviedo |

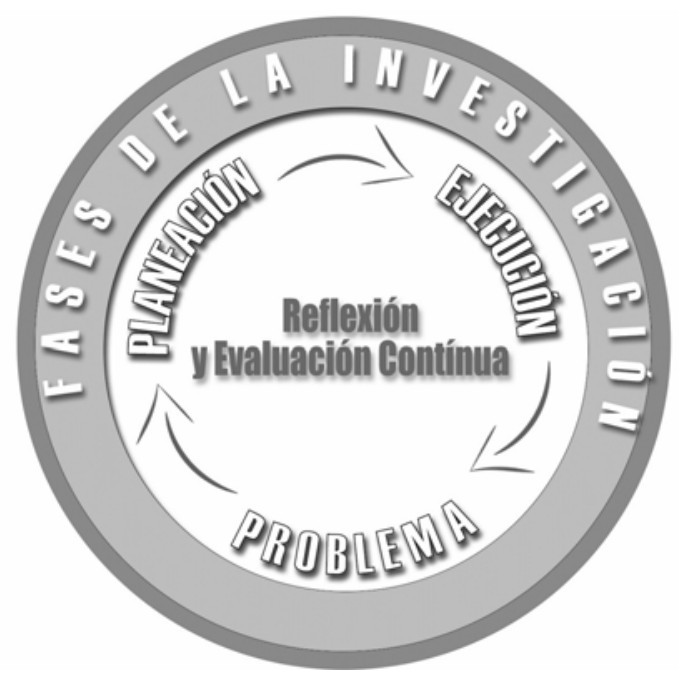

Fuente: elaboración propia

En este sentido, David Perkins (1997) indica que cuando un docente actúa de manera reflexiva, influye en el aprendizaje del estudiante. Por ello, el profesor debe tener en cuenta que la reflexión y evaluación continua no precisa un número de ciclos necesarios. En consecuencia, el tiempo constituye uno de los factores esenciales que el investigador debe tener en cuenta en el cambio, la transformación y la construcción de conocimiento.

Las fases de la Investigación que se tuvieron en cuenta para la realización de este proyecto fueron:

\section{PRIMERA FASE: EL PROBLEMA}

Esta fase comenzó con la identificación del problema, profundizando en la comprensión (diagnóstico), reflexión e interpretación de lo que sucedía desde el punto de vista de quienes actuaban e interactuaban en la situación. En correspondencia con lo anterior, se conformó y consolidó -con sus respectivas funciones y compromisos- la Comisión de evaluación y aprendizaje continuo (CAEC). Fue un grupo que estudió y reflexionó permanentemente sobre sus prácticas, para construir y mejorar los procesos pedagógicos, lograr transformación y conocimiento en las prácticas pedagógicas, mediante la interacción.
SEGUNDA FASE: PLANEACIÓN

Al haber reflexionado en el problema, se adoptó una postura teórica para conseguir una comprensión del problema. Los investigadores y actores de la CAEC, formularon el sistema de preguntas y objetivos de la investigación, construyeron un plan que incluyó la revisión del diagnóstico y sus hallazgos; además, plantearon un conjunto de acciones, con el fin de atender a las situaciones encontradas. Igualmente, podía ser la reconstrucción de la estrategia. Conocidas las falencias, se hizo posible incursionar en el diseño de una práctica nueva, por consiguiente, el maestro entraba a rediseñar la planeación con una nueva práctica más efectiva que tenía que ver con la validación de los procesos. De igual manera, planearon un cronograma, indicadores de resultados, responsables, evidencias con sus respectivas fuentes de verificación y, por último, un presupuesto.

\section{TERCERA FASE: EJECUCIÓN}

Se interpretó lo que ocurrió, teniendo en cuenta la observación, flujo de información y diálogo libre de trabas; los registros de la práctica aseguraron el cumplimiento de las actividades en los tiempos establecidos; el seguimiento permitió reflexión y evaluación continua, la validación de la efectividad de la práctica y el paso a los

ciclos siguientes en caso de haber sido necesario. Todas las fases se monitorearon comprobando la efectividad de los indicadores y los objetivos.

\section{DEFINICIÓN DE LAS CATEGORÍAS}

No se establecieron categorías previas a la investigación. Estas surgieron desde la identificación del problema y la formulación de los objetivos, mediante la participación de los actores, la observación, diálogo libre, los registros escritos, narraciones y diarios de campo.

El sistema de categorización construido cumple con las características de las categorías propuestas por Rodríguez, Gil y García (1996), a saber: las unidades diferenciadas de los datos se incluyen en una sola categoría, ordenadas y clasificadas; son claras para cada uno de los codificadores relevantes respecto a los objetivos del estudio y adecuadas al contenido analizado. 
Figura 3. Diseño de categoría de análisis.

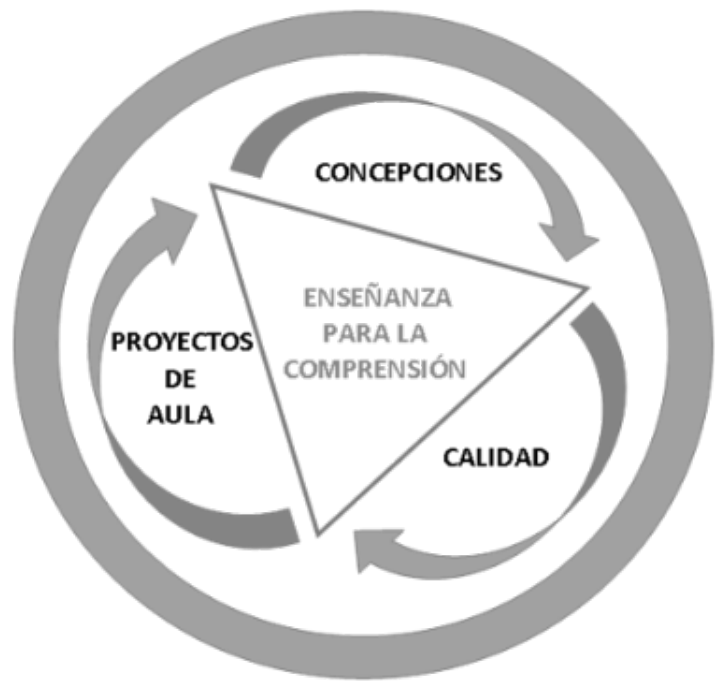

Fuente: elaboración propia

Los proyectos de aula: son estrategias didácticas que tienen por objeto identificar alternativas de enseñanza y aprendizaje por medio de la interacción entre el maestro, el estudiante y el contenido. En este sentido, Sayago $Z$ (2003) argumenta que los proyectos de aula desencadenan procesos de cuestionamientos e identificación de problemas de una manera entretenida, generados por los estudiantes y las propuestas expuestas por el docente mediante las actividades.

Concepciones: de acuerdo con la investigación, se ha entendido que una concepción tiene que ver con el punto de vista de las personas. Para Van Driel (2007), una propuesta didáctica combina concepciones educativas en función del contexto, enfocada a desarrollar las habilidades y actitudes de los estudiantes. En este sentido, se aprende haciendo y practicando (Fernández, Portela, González y Elortegui, 2001). Por lo tanto, en la enseñanza se consideran las concepciones previas de los estudiantes, intereses y necesidades, se conectan los conocimientos con su vida cotidiana y se fomenta el desarrollo de habilidades.

Calidad educativa: entendemos que una educación de calidad es aquella que forma mejores seres humanos, ciudadanos con valores éticos, respetuosos de lo público, que ejercen los derechos humanos, cumplen con sus deberes y conviven en paz. Una educación que genera oportunidades legítimas de progreso y prosperidad para ellos y para el país. Para Graells (2002), la calidad en la educación asegura a todos los estudiantes la adquisición de los conocimientos, capacidades, destrezas y actitudes necesarias para la vida.

Tomando como referencia lo anterior, se desarrolló un sistema de objetivos y categorías conceptuales con el propósito de detallar minuciosamente la relación y alcance de estas con la investigación presentada.

\section{RESULTADOS}

Los resultados de la investigación se dieron acorde con los objetivos específicos, y en correspondencia con las fases de la investigación acción definidas en la metodología, una mirada reflexiva sobre las experiencias docentes. El término experiencias está referido a las prácticas didácticas de los docentes utilizando el modelo educativo Enseñanza para la comprensión y los proyectos de aula.

En forma colectiva y participativa de los docentes del colegio Visión Mundial, se logró consolidar la Comisión de aprendizaje y evaluación continua - CAEC, con el fin de indagar, explorar, reflexionar y de manera creativa formar círculos de aprendizaje y adquirir nuevos conocimientos. De igual forma, se definieron los diferentes compromisos compartidos para la realización de la investigación. En este caso, los integrantes de la Comisión lograron un vínculo de interacción y de reconocimiento, se generaron algunas inquietudes y cuestionamientos frente a los proyectos de aula, el modelo pedagógico Enseñanza para la comprensión, las prácticas docentes y la calidad educativa, problemas comunes en el proceso de enseñanza y aprendizaje. De igual forma, se identificó y analizó el problema, se trabajó alrededor del mismo, considerando las ideas expuestas, diferencias generadas y las diversas propuestas de acuerdo con los criterios conceptuales unificados previamente.

En este sentido, la Comisión de aprendizaje y evaluación continua (CAEC), se constituyó en una contribución pedagógica y se convirtió en una unidad integradora, donde la docencia, la investigación y la práctica real fueron elementos básicos en el proceso de la investigación acción; se centraron en la observación del contexto en el aula y la escuela, en sus propias prácticas de manera real y espontánea, realizaron trabajo de campo en la medida que el maestro investigador recogía información atendiendo a su doble rol como sujeto observado y sujeto
| La investigación

acción y el

aprendizaje por

proyectos en el

marco del modelo

pedagógico

Enseñanza para

la comprensión.

Experiencia del

colegio Visión

Mundial en

comunidades

vulnerables de

Montería.
| Panorama

| pp. 39-51

| Volumen 11

| Número 21

| Julio-Diciembre | 2017 
PREGUNTAS Y OBJETIVOS

Martha Chaves

Silva

Lilia Barrios

Oviedo |

¿Cuál es el impacto de los proyectos de aula como estrategia didáctica integradora en la dinamización de los procesos de enseñanza y aprendizaje?
Analizar el impacto de los proyectos de aula, como estrategia didáctica integradora en la dinamización de los procesos de enseñanza y aprendizaje.
CATEGORÍAS CONCEPTUALES

OBJETIVOS

ESPECÍFICOS

CATEGORIAS

RASGOS

DESCRIPTORES
Comprender las

de los docentes, estudiantes y

padres de familia del colegio

Visión Mundial, en relación con las prácticas de enseñanza y aprendizaje en el marco del modelo pedagógico

Enseñanza para la comprensión.

Saberes de
los docentes,
estudiantes
y padres de
familia frente a
la enseñanza y
el aprendizaje
en el modelo
EpC y la
investigación
acción.

Concepciones acerca del modelo EpC

Concepciones acerca de los proyectos de aula

Concepciones de las prácticas de enseñanza y aprendizaje

$\begin{array}{cc}\text { ¿Qué valoraciones } & \text { Valorar los } \\ \text { amerita el impulso } & \text { resultados de } \\ \text { de los proyectos } & \text { los proyectos de } \\ \text { de aula en relación } & \text { aula en relación } \\ \text { con el desarrollo } & \text { con el desarrollo } \\ \text { académico, personal } & \text { académico, personal } \\ \text { y sociocultural de } & \text { y sociocultural } \\ \text { los miembros de la } & \text { de los miembros } \\ \text { comunidad educativa? } & \text { de la comunidad } \\ & \text { educativa. }\end{array}$

Panorama pp. 39-51 |

Volumen 11 |

Número $21 \mid$ exploratoria, produciendo descripciones importantes para la investigación, reconociéndole a cada maestro la capacidad de reunir y registrar libremente las manifestaciones intersubjetivas de los sujetos enfrentados a

Fuente: elaboración propia.

observador para comprender y transformar la realidad. De la misma forma, el trabajo de campo, la observación y el registro escrito de lo observado fueron instrumentos que permitieron recoger información de la experiencia sus contextos reales, asumiendo una actitud de crítica y
Metodologías

de clase

innovadoras

Estrategias

didácticas

integradoras

AULA

enseñanza por proyecto en las prácticas educativas

Prácticas educativas inclusivas y transformadoras
Impacto en la comunidad educativa

Practica de valores

CALIDAD
Aportes académicos, sociales y personales institucionales

Transformación social

Resultados académicos satisfactorios de autocrítica que en síntesis surgieron a interrogantes de carácter teórico y conceptual con fines de transformación, innovación, reflexión y evaluación continua, producción intelectual y el diseño de propuestas para el mejoramiento de las prácticas de enseñanza. En este sentido, para Briones (2000), el maestro se compromete directamente con la investigación como función de su labor como educador con los siguientes ejes articuladores: 
Reflexivo con la investigación-acción en su práctica diaria, con capacidad de desarrollar teoría de sus actividades y pruebas para la acción, generando innovación en los diversos ciclos entre la acción y la teoría. Para Carr y Kemmis (1986), "ciclos de investigación-acción, este círculo virtuoso convierte la práctica en un círculo creativo de reflexión, analizando cada una de las partes de la acción como un todo" (p. 197).

Participante, protagonista y desarrollador de la investigación, en un proyecto de colaboración con capacidad de iteración. La realidad del aula y de la institución se construye por los que en ella están integrados. La iteración aquí es entendida como una dinamización, retroalimentación y transformación, es el proceso cíclico (reflexión-evaluación continua), incorporando a la reflexión el desarrollo de valores y principios educativos.

Beneficiario de los resultados de la investigación educativa, con capacidad de construir y transformar a partir de la realidad, con la posibilidad de desarrollar estrategias para interpretar y reflexionar sobre la práctica. Recoger otros puntos de vista, además de los propios. Confrontar diferentes perspectivas sobre la misma situación y usar las diferencias como punto de partida para el desarrollo de una teoría de la práctica.

Sistematiza sus experiencias y reflexiones pedagógicas, con capacidad de producción académica generando cambios en las prácticas didácticas y formativas que se está requiriendo y apunta hacia la calidad educativa, de tal manera que esta sea asumida como un centro de investigación y desarrollo de las diferentes formas de la subjetividad humana (Rodríguez, 2000).

El siguiente esquema, es el diseño de los ejes articuladores de los integrantes de la CAEC.

Figura 3. Diseño de ejes articuladores de la Comisión de aprendizaje y evaluación continua.

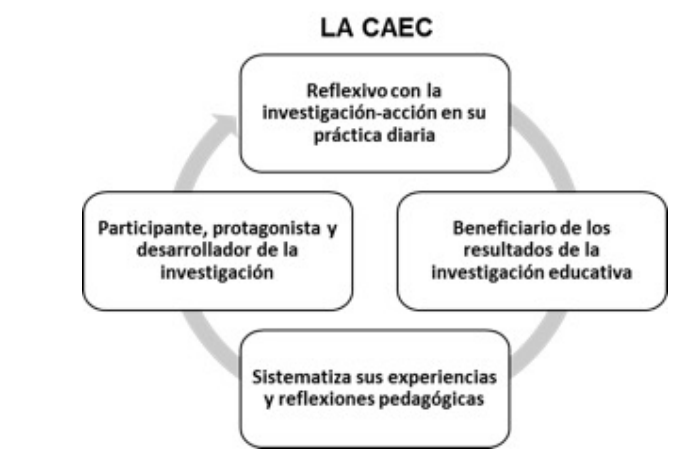

Fuente: elaboración propia
Las concepciones, los proyectos de aula y la calidad educativa de la práctica docente mostraron la relación directa entre la formación de los maestros, los logros de los estudiantes, la incidencia de las familias, sin embargo, consideraron que están determinadas por el contexto social.

Las reflexiones aportadas por los docentes reconocen los proyectos de aula para hacer innovación en el salón de clase, construir alternativas que propicien aprendizajes más significativos y pertinentes, analizando el contexto en que viven los estudiantes,

Algunos de los estudiantes valoran las prácticas educativas y lineamientos académicos desarrollados en la institución, destacando el interés y motivación que se promueve constantemente en el aula de clase. A nivel general, se evidencia que los estudiantes no reconocen el modelo EpC, Enseñanza para la comprensión, desde sus dimensiones teórico pedagógicas; pero sí valoran la praxis que desarrollan sus maestros.

El colegio ha incentivado y permitido la integración de los padres, madres y acudientes en la acción educativa. Inclusive, se visibiliza con mayor preponderancia la participación de los padres de familia en el proceso de enseñanza y aprendizaje, ratificando que la familia es el ámbito natural en el desarrollo de los niños.

\section{CONCLUSIONES}

La investigación educativa como posibilitadora de construcción de conocimiento se convierte en instrumento para descubrir el mundo complejo de la escuela. La investigación como base de la enseñanza y de formación permite al docente, desde la reflexión, la construcción de saber, sin embargo, como bien lo ha señalado el profesor Porlán (1995), solo una reflexión que incorpore la crítica ideológica (citando a Carr y Kemmis, 1986) puede revelar a los educadores ilusiones ideológicas que ayudan a preservar un orden social ajeno a sus experiencias y necesidades colectivas. El ejercicio investigativo puede dirigir procesos reales de transformación, no solo de las prácticas pedagógicas sino de las prácticas sociales inmersas en la escuela, favoreciendo la democratización del conocimiento y atenuando los efectos de la desigualdad social.
La investigación

acción y el

aprendizaje por

proyectos en el

marco del modelo

pedagógico

Enseñanza para

la comprensión.

Experiencia del

colegio Visión

Mundial en

comunidades

vulnerables de

Montería.
| Panorama

| pp. 39-51

| Volumen 11

| Número 21 
Martha Chaves

Silva

Lilia Barrios Oviedo académicos de los estudiantes, el impacto, los cambios y las transformaciones en la comunidad educativa.

Los procesos académicos parten del modelo pedagógico Enseñanza para la comprensión, EpC; este modelo mira hacia el estudiante, lo reconoce como un sujeto con voz, con conciencia y, sobre todo, con inteligencia, en este viraje, el sentido de la formación tiene que ver con el desarrollo de habilidades y competencias en el estudiante, como una apuesta de reconstrucción de lo humano en el marco de la complejidad de lo social.

En consecuencia, el proyecto de aula como herramienta didáctica es una apuesta a optimizar los procesos de enseñanza - aprendizaje, diseñando e implementando acciones que impulsen el logro de los estudiantes, y que se constituyan en mecanismos esenciales para el mejoramiento continuo.

Los estudiantes, con la orientación de los docentes, pueden identificar puntos fuertes y débiles en el desarrollo de sus competencias para la vida, y participar así de los procesos de mejoramiento continuo y de evaluación en el aula. Igualmente, los proyectos de aula fomentan los canales de comunicación entre los distintos miembros de la comunidad educativa (docentes, directivos, estudiantes, familias), y hace que se compartan metas educativas comunes, relacionadas con una formación integral y con la adquisición de las competencias necesarias para desempeñarse efectivamente en la sociedad.

Panorama pp. 39-51|

Volumen 11 | Número 21 | Julio-Diciembre | 2017 | argumentos y criterios para interactuar con las distintas actividades organizadas por los docentes en torno a la formación de los estudiantes.
Después de haber llevado a cabo el proyecto de investigación, los maestros identificaron la importancia de integrarle a la práctica educativa la reflexión. La investigación-acción no se constituye solo como un ejercicio individual, sino que está inmersa en las complejas interacciones entre el docente, los estudiantes, padres de familia, el contexto social, y de las relaciones entre pares que se desarrollan en los entornos educativos. Para Sacristán y Pérez Gómez (1992) es rescatar esas distancias existentes como medio para entender los procesos pedagógicos y, sobre todo, al enfrentarnos con los problemas de la calidad educativa, los cambios y la transformación de las prácticas escolares y el impacto y la transformación del mismo contexto social.

También resulta interesante demostrar que la educación de calidad para poblaciones en condiciones de pobreza es no solo necesaria, sino posible, a pesar de que los recursos económicos sean iguales o inferiores a los que asigna el Estado a cualquier institución educativa pública, aminorando la fuerte estratificación que persiste en el sistema educativo local.

En este orden de ideas, para mantener y enriquecer esta calidad, es necesario incrementar el apoyo a la actividad de investigación en educación y pedagogía. Se requieren esfuerzos permanentes, sistemáticos e institucionalizados de investigación sobre educación, con el fin de consolidar una comunidad y un pensamiento estratégico en el tema, lograr una socialización amplia en la sociedad colombiana de la apropiación del conocimiento que se genera y su traducción hacia el campo de la adopción por parte, tanto de las políticas públicas, como de las políticas institucionales de las entidades que conforman el sistema de educación.

Finalmente, tomando como referencia las categorías conceptuales, se evidencia el impacto del modelo Enseñanza para la comprensión y la Enseñanza por proyectos, en los escenarios educativos que viven los estudiantes en sus procesos de aprendizaje, reconociendo el papel que ha jugado la escuela y los maestros en la consolidación de sujetos con voz y voto capaces de tomar decisiones consecuentes con las dinámicas de la sociedad. De igual forma, se reitera la transformación social y ética que ha liderado el colegio en sus familias, reflejada en el comportamiento y aspiraciones de sus hijos. 


\section{REFERENCIAS}

1. Briones, G. (2000). Evaluación de programas sociales. México: Trillas.

2. Bru, M. y Basagoiti. M. (2002). La InvestigaciónAcción Participativa como metodología de mediación e integración socio-comunitaria. Recuperado de: http://www.pacap.net/es/publicaciones/pdf/ comunidad/6/documentos_investigacion.pdf

3. Carr, W.y Kemmis, S. (1986). Teoría critica de la enseñanza. Barcelona: Martínez Roca.

4. Clark. C. y Peterson, P. (1990). Procesos de pensamiento de los profesores. En M. Wittrock (dir.), La investigación de la enseñanza III. Profesores y alumnos (443-543). Barcelona: Paidós.

5. Coll, C. (1981) Algunos problemas planteados por la metodología observacional: niveles de descripción e instrumentos de validación. Anuario de Psicología, 24(1), 111-131.

6. Denegrí, M. (1996) Las ideas de Piaget y Vigotsky. Santiago de Chile: Editorial La Salle.

7. Denegrí, M. (1996) Desarrollo y aprendizaje: la ideas de Piaget y Vigotsky en el aula. Santiago de Chile: Editorial La Salle.

8. Denegrí, M. (2000) Orientaciones pedagógicas constructivistas. Unidad de producción de materiales educativos. EDUCADE Consultora Ltda.

9. Denegrí, M. y Martínez, G. (2001). Proyectos de aula interdisciplinarios: una mirada desde la psicología educacional. En Psicología y Educación. Encuentros y desencuentros. Juliá, M. y Catalán, J. (Eds.) Departamento de Publicaciones Universidad de La Serena.

10. Elliott, J. (1993). El cambio educativo desde la investigación-acción. Madrid: Morata S.L

11. Fernández, J., Portela, L., González, B.y Elortegui, N. (2001). Las analogías en el aprendizaje de la física en secundaria. Comunicación presentada en el I Congreso Nacional de Didácticas Específicas. Las Didácticas de las Áreas Curriculares en el siglo XXI. Granada.

12. Graells, M (2000)."Criterios de calidad en los programas educativos". Revista MasPC, (8), 218-219.

13. Guzmán, B. y Jiménez, P. (1991). "El aula: espacio de interrelación de quehaceres y finalidades educativas", en: El aula universitaria, UNAM, México, 338.

14. Hernández, F. y Ventura, M. (1994). La organización del curriculo por proyectos de trabajo. Barcelona: Editorial Grao.

15. Kaplan, A. (1979). The conduct of inquiry: methodology for behavioral sciences. New York: Harper.
16. Kemmis, S. (1992). El currículum: va más allá de la teoria de la reproducción. Madrid: Morata.

17. Kemmis, S. y McTaggart, R. (1992). Cómo planificar la investigación-acción. Barcelona: Laertes

18. Kemmis, S. y McTaggart, R. (1998). The Action Research Planner. Australia: Deakin University.

19. LeCompte, M., Millroy, W.y Preissle, J. (Eds.). (1992). The handbook of qualitative research in education. California: Academic Press.

20. Pérez, G. (1994). Investigación cualitativa. Retos e interrogantes. Madrid: La Muralla.

21. Perilla, L. y Rodríguez E. (2010). Proyectos de aula: una estrategia didáctica hacia el desarrollo de competencias investigativas. Uniminuto Regional Villavicencio: Educrea.

22. Perkins, D. (1995). La escuela inteligente. Del adiestramiento de la memoria a la educación de la mente. Barcelona: Ed. Gedisa.

23. Perkins, D. (1997). Del adiestramiento de la memoria a la educación de la mente. Barcelona: Editorial Gedisa. Barcelona.

24. Perkins, D. (1999). ¿Qué es la Comprensión? Revista Paidós. 01-04.

25. Pogré, P. (2007). ¿Cómo enseñar para que los estudiantes comprendan? Revista Diálogo Educacional, 7(20), 25-32.

26. Porlán, R. (1995). Constructivismo y Escuela. Sevilla: Díada.

27. Restrepo, B. (2007). Investigación acción y educación. Ponencia presentada en el Simposio Internacional de Investigación Acción y Educación en contexto de pobreza. Bogotá: Universidad de la Salle.

28. Rodríguez, G., Gil, F. y García, J. (1996). Metodología de la investigación cualitativa. Granada: Aljibe.

29. Rodríguez, S. (2000). El Proyecto de Aula como Estrategia Pedagógica. Universidad de la Sabana.

30. Sandín, M. (2003). Investigación Cualitativa en Educación. Fundamentos y Tradiciones. Madrid: McGraw Hill.

31. Stenhouse, L. (1984). La investigación como base de la enseñanza. Madrid: Ediciones Morata.

32. Van Driel, J., Bulte, A. y Verloop, N. (2007). The relationships between teachers' general beliefs about teaching and learning and their domain specific curricular beliefs. Learning and Instruction, (17), 156-171.

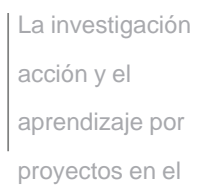

marco del modelo

pedagógico

Enseñanza para

la comprensión.

Experiencia del

colegio Visión

Mundial en

comunidades

vulnerables de

Montería. 